\title{
Clinical features and the degree of cerebrovascular stenosis in different types and subtypes of cerebral watershed infarction
}

Yue Li $i^{1}$, Man Li ${ }^{2}$, Xiaoyu Zhang ${ }^{3}$, Shuna Yang ${ }^{1}$, Huimin Fan ${ }^{1}$, Wei Qin ${ }^{1}$, Lei Yang ${ }^{1}$, Junliang Yuan ${ }^{1}$ and Wenli Hu ${ }^{1 *}$

\begin{abstract}
Background: Whether there are differences in pathogenesis among different types and subtypes of cerebral watershed infarction (WSI) is controversial since they have been combined into a single group in most previous studies.

Methods: We prospectively identified 340 supratentorial WSI patients at Beijing Chao-Yang Hospital, Capital Medical University, China and classified them based on diffusion-weighted imaging(DWI) templates. Baseline characteristics, clinical courses and neuroradiological features were compared among patients with different types and subtypes of WSI.

Results: We identified 92 patients with cortical watershed infarction (CWI), 112 with internal watershed infarction (IWI) and 136 with mixed-type infarction. Compared with CWI patients, more IWI patients had critical stenosis of internal carotid artery (ICA) $(P<0.001)$. For the CWI group, patients with anterior watershed infarction (AWI) were more prone to critical ICA stenosis than those with posterior watershed infarction (PWI) $(P=0.011)$. For the IWI group, critical ICA stenosis was more prevalent in patients with partial IWI (P-IWI) than in those with confluent IWI (C-IWI) $(P=0.026)$. IWI patients were more frequently found to have clinical deterioration during the first 7 days of hospitalization and a poor prognosis at the 90th day than in CWI patients ( $P=0.003$ and $P=0.014$, respectively).
\end{abstract}

Conclusions: IWI, especially the P-IWI subtype, is associated with hemodynamic impairment (HDI), whereas CWI has a weaker correlation with ICA steno-occlusion. Furthermore, IWI patients are more prone to poor prognosis.

Keywords: Cerebral watershed infarction, cerebrovascular stenosis, hemodynamic impairment, clinical features, prognosis

\section{Background}

Cerebral watershed infarction (WSI), ischemic lesions between two non-anastomosing main arterial territories, can be classified as either cortical watershed infarction (CWI; or external watershed infarction) or internal watershed infarction (IWI; or subcortical watershed infarction), both of which can be further divided into subtypes. The mechanism of WSI has not been fully understood. Traditionally, hemodynamic impairment

\footnotetext{
* Correspondence: huwenli@sina.com

${ }^{1}$ Department of Neurology, Beijing Chao-Yang Hospital, Capital Medical

University, No. 8 South Gongti Road, Chaoyang district, Beijing 100020, China

Full list of author information is available at the end of the article
}

(HDI) has been widely accepted as a cause of WSI [1], but microemboli may also contribute to it [2].

It is unclear whether the mechanism differs among different types and subtypes of WSI since most previous studies either combined CWI and IWI into a single group or focused only on one type or subtype of WSI. Only a few studies [3-5] tried to find the differences in the characteristics of different WSI types but yielded controversial results. Therefore, whether the pathogenesis differs among them is unclear. In addition, cases where both CWI and IWI are present, which are named mixed-type infarction in our study, have never been included in previous studies. 
In this study, we tried to distinguish the mechanisms of different types and subtypes of WSI. Based on the analysis of pre-existing results, we postulated that IWI was more relevant to HDI than CWI. Clinical courses and short-term clinical outcomes were also compared among different types of WSI.

\section{Methods}

\section{Subjects}

Patients with acute ischemic stroke admitted to the neurology department of Beijing Chao-Yang Hospital, Capital Medical University from August 2013 to January 2016 were identified. Only patients who met the following criteria were recruited: (1) admitted to the hospital within 7 days of symptom onset; (2) identified as WSI based on diffusion-weighted imaging(DWI) sequence; (3) completed evaluations including medical history, risk factors, cardiologic test, routine blood tests, stroke scales and cerebrovascular examinations. Potential sources of cardioembolism (PSCE) were identified if any of the following existed: recent myocardial infarction ( $<3$ weeks), atrial fibrillation, dilated cardiomyopathy, acute bacterial endocarditis, mitral stenosis, prosthetic valve replacement, sick sinus syndrome or patent foramen [6].

\section{Ethics}

Informed consent was obtained from patients for magnetic resonance imaging (MRI) and computed tomographic angiogram (CTA), and to the use of data for research. All medical procedures were performed only for clinical reasons. The study was approved by the Ethics Committee of Beijing Chao-Yang Hospital, Capital Medical University and was in accordance with the declaration of Helsinki.

\section{Imaging protocol}

MRI was performed on the same 3.0 T Siemens scanner (Erlangen, Germany). The parameters of MR examination were as follows: axial T2-weighted (repetition time, $4500 \mathrm{~ms}$; echo time, $93 \mathrm{~ms}$ ), axial T1-weighted imaging (repetition time, $2000 \mathrm{~ms}$; echo time, $9.2 \mathrm{~ms}$ ), axial diffusion-weighted imaging (repetition time, $3300 \mathrm{~ms}$; echo time, $91 \mathrm{~ms}$ ), and coronal fluid-attenuated inversion recovery sequences (repetition time, $8000 \mathrm{~ms}$; echo time, $86 \mathrm{~ms}$ ).

CTA was performed on a dual-source CT scanner (Somatom Definition; Siemens Medical Solutions, Forchheim, Germany). For head and neck CTA, 70-80 ml non-ionic contrast agent (350 $\mathrm{mg}$ iodine $/ \mathrm{ml}$ ioversol, Optiray 350, Mallinckrodt Pharmaceuticals) was injected into the antecubital vein at $5 \mathrm{ml} / \mathrm{s}$ followed by a $40-\mathrm{ml}$ saline flush also at $5 \mathrm{ml} / \mathrm{s}$. The CTA covered the area from just below the aortic arch to the vertex. CTA was performed in a helical scan mode using the following parameters: $64 \times 0.6$ collimation, 0.65 pitch, $120 \mathrm{kV}$, automatic exposure control with standard deviation of 10 and exposure range $100-700 \mathrm{~mA}, 0.625 \mathrm{~mm}$ and $3.0 \mathrm{~mm}$ slice thickness, $0.33 \mathrm{~s}$ rotation time, reconstruction filter FC43 and standard AIDR3D. The bolus tracker was set at an absolute threshold of $180 \mathrm{HU}$ at the level of the descending aorta in dual energy mode.

\section{MRI imaging analysis}

Two experienced neurologists (Lei Yang and Man Li) who were unaware of the patient's clinical information typed the classification with templates [7]. CWI was defined as hyperintense areas on DWI sequence in the junctions of the anterior cerebral artery (ACA), middle cerebral artery (MCA), and posterior cerebral artery (PCA) territories, as a thin fronto-parasagittal wedge. CWI was further divided into anterior watershed infarction (AWI, between ACA and MCA), posterior watershed infarction (PWI, between MCA and PCA) and both-type infarction (AWI plus PWI). IWI was defined as hyperintense areas on DWI sequence between the deep and superficial perforating arteries of the MCA, and further divided into partial IWI (P-IWI, a single lesion or a chain-like, the so-called "rosary-like" pattern in the centrum semiovale) and confluent IWI (C-IWI, large cigar-shaped infarction alongside the lateral ventricle). A concurrence of CWI and IWI was identified as mixed-type infarction. Classifications of WSI, CWI and IWI is presented in Fig. 1.

We used North American Symptomatic Carotid Endarterectomy Trial (NASCET) method to measure the degree of cerebrovascular steno-occlusion as [8]: non-significant $(<50 \%)$, moderate $(50 \%$ to $74 \%)$, severe (75\% to $99 \%$ ), or occluded. The critical stenosis was defined as $>75 \%$ stenosis or occlusion. The $\mathrm{k}$-coefficient for interobserver agreement was 0.824 for the classification of WSI and 0.797 for the degree of cerebrovascular steno-occlusion.

\section{Demographic and clinical assessment}

The following clinical information was collected: sex, age, history of hypertension (defined as treatment with antihypertensive medications or BP > 140/90 $\mathrm{mmHg}$ measured on several separate occasions), diabetes mellitus (defined as treatment with antidiabetic medications or fasting plasma glucose $>7.0 \mathrm{mmol} / \mathrm{L}$ ), hyperlipidemia (based on history), smoking (continuously smoking $>1$ cigarette a day for 6 months), stroke or transient ischemic attack (TIA) and coronary artery disease (CAD, defined as a history of myocardial infarction or angina pectoris), blood pressure (BP, recorded at admission) and results of laboratory tests including level of total cholesterol (TC), triglyceride (TG), high density lipoprotein (HDL), low density lipoprotein (LDL), glycosylated 
a

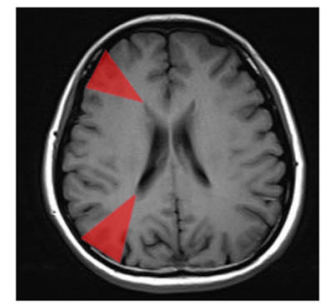

Cortical Watershed Infarction (CWI)

b

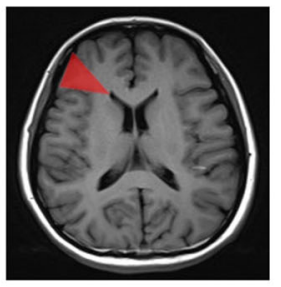

Anterior Watershed Infarction (AWI)

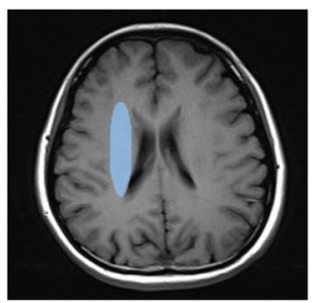

Internal Watershed Infarction (IWI)

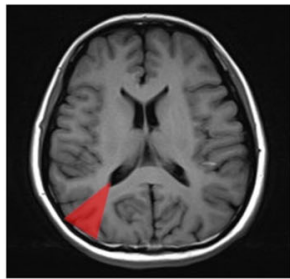

Posterior Watershed Infarction (PWI)

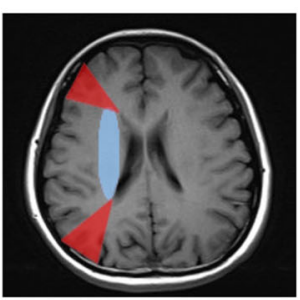

Mixed-type

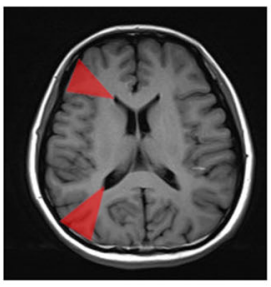

Both-type

C



Partial IWI (P-IWI)



Confluent IWI (C-IWI)

Fig. 1 Classification of watershed infarction (WSI): (a) types of WSI; (b) subtypes of cortical watershed infarction (CWI); (c) subtypes of internal watershed infarction (IVI)

hemoglobin (HbA1c), albumin, plasma fibrinogen (Fib), homocysteine ( $\mathrm{HCY})$, high-sensitivity $\mathrm{C}$-reactive protein (hs-CRP), serum creatinine ( $\mathrm{Cr}$ ) and uric acid (UA).

All patients received standard treatment in the stroke unit. The National Institutes of Health Stroke Scale (NIHSS) score was checked at day 1 and 7 after admission. The clinical course was defined by change of NIHSS score between the first and seventh day after admission as: 1) improved (NIHSS score decreased by $\geq 2$ points); 2) stable (NIHSS score decreased by $<2$ points); and 3) deteriorated (NIHSS score increased after admission). Short-term clinical outcome was assessed by Modified Rankin Scale (mRS) 3 months after stroke onset via telephone follow-up and we defined poor outcome as mRS score $>3$ points.

\section{Statistical analysis}

All statistics were presented as mean \pm standard deviation (SD) for continuous variables with normal distribution, median and interquartile range for continuous variables with non-normal distribution, and counts and proportions for categorical variables. Differences among the groups and subgroups were examined using Pearson $x^{2}$ test, one-way analysis of variance (ANOVA), or non- parametric test, depending on the nature of the variables being compared. The Bonferroni correction was used for post hoc comparisons to obtain an adjusted significance level for each domain-specific test: $\approx 0.05 / 3=0.017$. Statistical significance was established at $P<0.05$. Logistic regression analysis was applied to identify independent predictors of poor clinical outcome. Independent variables were age, sex, vascular risk factors, PSCE, clinical course, NIHSS score at admission, type of WSI, and the critical stenosis of ICA and MCA. The results were presented as estimates of relative risk by odds ratio (OR) with a 95\% CI. Analysis was performed with Statistical Package for Social Sciences (SPSS version 24).

\section{Results}

\section{Participants characteristics}

During the study, 351 WSI patients were identified, among whom 11 with incomplete examinations were excluded. As a result, 340 patients were recruited with $220(64.70 \%)$ males. Among the 340 WSI patients, 92 (27.10\%) had CWI, 112 (32.90\%) had IWI and 136 (40.00\%) had mixed-type infarction. Details of clinical and demographic features of the patients in each group 
Table 1 Clinical and demographic features of patients with different types of WSI

\begin{tabular}{|c|c|c|c|c|}
\hline Variable & $\begin{array}{l}\text { CWI } \\
(n=92)\end{array}$ & $\begin{array}{l}|\mathrm{W}| \\
(n=112)\end{array}$ & $\begin{array}{l}\text { Mixed-type } \\
(n=136)\end{array}$ & $P$ \\
\hline Male, $n(\%)$ & $54(58.7)$ & $73(65.2)$ & $93(68.4)$ & 0.321 \\
\hline Age, years & $65.46 \pm 10.42$ & $66.12 \pm 10.89$ & $64.54 \pm 11.26$ & 0.429 \\
\hline \multicolumn{5}{|l|}{ Risk factors, $n(\%)$} \\
\hline Hypertension & $60(65.2)$ & $79(70.5)$ & $93(68.4)$ & 0.718 \\
\hline Diabetes mellitus & $27(29.3)$ & $36(32.1)$ & $43(31.6)$ & 0.903 \\
\hline Hyperlipidemia & $48(52.2)$ & $60(53.6)$ & $63(46.3)$ & 0.480 \\
\hline PSCE & $22(23.9)$ & $14(12.5)$ & $20(14.7)$ & 0.071 \\
\hline Smoking & $53(57.6)$ & $72(64.3)$ & $82(60.3)$ & 0.613 \\
\hline Stroke / TIA & $28(30.4)$ & $38(33.9)$ & $52(38.2)$ & 0.468 \\
\hline$C A D$ & $21(22.8)$ & $15(13.4)$ & $16(11.8)$ & 0.059 \\
\hline $\mathrm{SBP}, \mathrm{mmHg}$ & $147.43 \pm 25.13$ & $154.45 \pm 23.07$ & $152.87 \pm 23.78$ & 0.791 \\
\hline $\mathrm{DBP}, \mathrm{mmHg}$ & $85.32 \pm 11.45$ & $87.23 \pm 12.87$ & $86.24 \pm 11.89$ & 0.613 \\
\hline \multicolumn{5}{|l|}{ Laboratory data } \\
\hline $\mathrm{TC}, \mathrm{mmol} / \mathrm{L}$ & $4.32 \pm 1.12$ & $4.69 \pm 0.93$ & $4.54 \pm 1.18$ & 0.512 \\
\hline $\mathrm{TG}, \mathrm{mmol} / \mathrm{L}$ & $1.23(0.92,1.89)$ & $1.49(0.98,2.16)$ & $1.37(1.02,2.09)$ & 0.897 \\
\hline $\mathrm{HDL}, \mathrm{mmol} / \mathrm{L}$ & $1.19 \pm 0.31$ & $1.36 \pm 0.27$ & $1.37 \pm 0.39$ & 0.318 \\
\hline $\mathrm{LDL}, \mathrm{mmol} / \mathrm{L}$ & $2.52 \pm 0.71$ & $2.69 \pm 0.67$ & $2.62 \pm 0.62$ & 0.634 \\
\hline $\mathrm{HbA} 1 \mathrm{c}, \%$ & $6.40(5.60,7.90)$ & $6.50(5.80,8.10)$ & $6.40(5.70,7.80)$ & 0.692 \\
\hline Albumin, g/L & $37.62 \pm 4.87$ & $37.66 \pm 4.14$ & $37.21 \pm 4.54$ & 0.941 \\
\hline Fibrinogen, mg/dl & $334.76 \pm 65.87$ & $326.86 \pm 71.23$ & $313.23 \pm 70.65$ & 0.327 \\
\hline $\mathrm{HCY}, \mathrm{mmol} / \mathrm{L}$ & $17.00(14.00,20.00)$ & $19.00(15.00,24.00)$ & $18.00(15.00,23.00)$ & 0.093 \\
\hline $\mathrm{Hs}-\mathrm{CRP}, \mathrm{mg} / \mathrm{L}$ & $\begin{array}{l}2.45(1.17,7.83) \\
(1.06,8.65)\end{array}$ & $2.54(1.21,6.87)$ & $2.64(1.15,5.45)$ & 0.432 \\
\hline $\mathrm{Cr}, \mu \mathrm{mol} / \mathrm{L}$ & $84.76(70.87,105.92)$ & $85.16(75.32,97.98)$ & $84.94(73.66,99.72)$ & 0.675 \\
\hline Uric acid, $\mu \mathrm{mol} / \mathrm{L}$ & $321.87 \pm 91.76$ & $319.76 \pm 98.65$ & $312.55 \pm 97.60$ & 0.821 \\
\hline
\end{tabular}

Data are presented as mean \pm standard deviation, median (interquartile range) or counts (\%)

WSI Watershed infarction, CWI Cortical watershed infarction, IWI Internal watershed infarction, N Number of persons, PSCE Potential sources of cardioembolism, TIA Transient ischemic attack, CAD Coronary artery disease, SBP Systolic blood pressure, DBP Diastolic blood pressure, TC Total cholesterol, TG Triglyceride, HDL High density lipoprotein, LDL Low density lipoprotein, $\mathrm{HbA1c}$ Glycosylated hemoglobin, $H C Y$ Homocysteine, $\mathrm{Hs}$ - $C R P$ High-sensitivity $\mathrm{C}$-reactive protein, $\mathrm{Cr}$ Serum creatinine

are presented in Table 1. The age, sex, risk factors and laboratory data did not differ among three groups.

\section{Incidence of critical cerebrovascular stenosis in different WSI types and subtypes}

The incidence of critical stenosis of internal carotid artery (ICA) differed among three groups of WSI $(P=0.001)$, but that of other cerebral vessels did not. In further comparison, more patients in the IWI group were found to have critical ICA stenosis than those in the CWI group $(43.80 \%$ vs. $19.60 \%$; $P<0.001$, Fig. 2). These results are presented in Additional file 1: Table S1.

Among 228 patients who had CWI (including 136 mixed-type patients), patients with AWI were more prone to critical ICA stenosis than those with PWI (39.40\% vs. $20.70 \% ; P=0.011$, Fig. 3 ). Among 248 patients who had IWI (including 136 mixed-type patients), critical ICA stenosis was more common in the P-IWI subgroup than in the C-IWI subgroup (44.10\% vs. $30.40 \% ; P=0.026$, Fig. 4).

\section{Differences in clinical course of hospitalization and prognosis at 90th day}

Although NIHSS scores at admission did not differ significantly among the groups, clinical course during the first week after admission was significantly different: only $13.0 \%$ of CWI patients deteriorated compared with $33.9 \%$ of IWI patients and $25.7 \%$ of patients who had mixed-type infarction. Further analysis showed that patients with IWI were more prone to deterioration than those with CWI $(P=0.003)$. Follow-up prognosis 3 months after stroke showed significantly more IWI patients with a poor outcome than CWI patients $(P=0.014)$. Details of the clinical course and prognosis of the 3 groups are presented in Table 2 . 


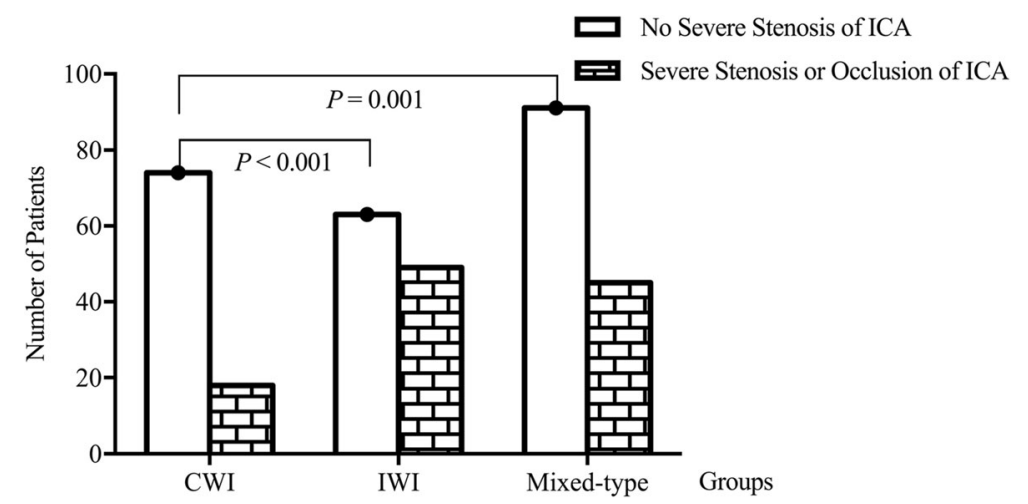

Fig. 2 Comparison of the incidence of critical internal carotid artery (ICA) stenosis among different types of WSI. The incidence of critical stenosis of ICA differed among three groups of WSI $(P=0.001)$, more patients in the IWI group were found to have critical ICA stenosis than those in the CWI group (43.80\% versus $19.60 \% ; P<0.001)$

After adjustment for confounding factors, multivariate logistic regression analysis revealed that the presence of IWI (OR, 5.231; 95\% CI, 1.687 to 9.703; $P=0.008$ ), critical stenosis of ICA (OR, 2.284; 95\% CI, 1.376 to 5.662; $P=0.012$ ), and the initial NIHSS score (OR, 1.877 ; $95 \% \mathrm{CI}, 1.176$ to $2.012 ; P=0.004$ ) were independently associated with poor outcome 3 months after stroke.

\section{Discussion}

In this study, we demonstrated that IWI, especially the P-IWI subtype, is associated with HDI and that IWI patients are more prone to poor prognosis. To our knowledge, this is the first study to investigate the mechanisms of different types and subtypes of WSI, for particular, the mixed-type infarction defined here as the concurrence of CWI and IWI.

Traditionally, WSI is mainly considered as a result of critical stenosis of main arteries, generally with precipitating factors such as acute hypotensive events, cardiopulmonary bypass and anesthesia. Another widely accepted hypothesis is that microemboli from the heart or artery-to-artery preferentially propagated to watershed areas because of their diameters [9].

Then, Caplan LR et al. [10] proposed a synergetic interaction theory: reduced perfusion weakens the ability to wash out microemboli, particularly within the borderzone areas. But another study [2] found that less microembolic signals (MES) were detected in patients with severe ICA stenosis and more in those with a lower degree of ICA stenosis. To explore the role of HDI and microemboli in the pathogenesis of WSI, Moustafa RR et al. [11] applied positron emission tomography and transcranial doppler in TIA/ minor stroke patients whose carotid stenosis was $\geq 50 \%$, and finally found that there was no significant difference between these two factors and no evidence of synergistic effect.

However, despite their inspirations, previous studies are limited in the following aspects. Firstly, CWI and IWI have been combined in most studies into a single group as a result of limited clinical cases. Some reports

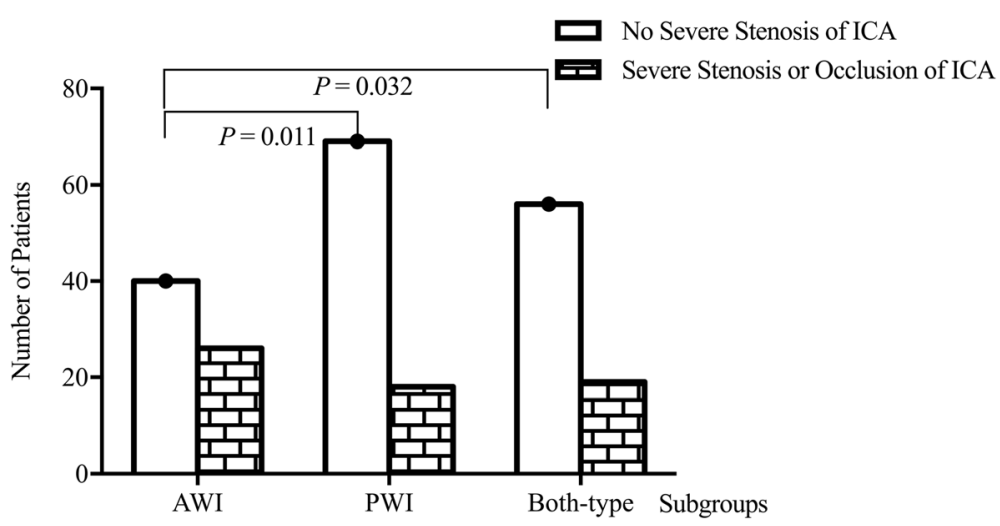

Fig. 3 Comparison of the incidence of critical ICA stenosis among different subtypes of CWI. The incidence of critical stenosis of ICA differed among three subgroups of CWI $(P=0.032)$, patients with AWI were more prone to critical ICA stenosis than those with PWI (39.40\% versus 20.70\%; $P=0.011)$ 


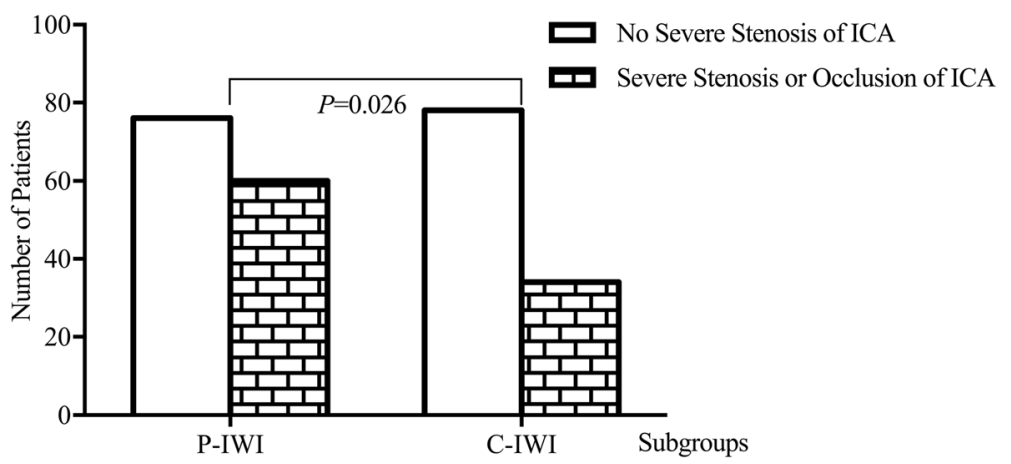

Fig. 4 Comparison of the incidence of critical ICA stenosis between different subtypes of IWI. Critical ICA stenosis was more common in the P-IWI subgroup than in the C-IVI subgroup ( $44.10 \%$ versus $30.40 \% ; P=0.026)$

focused on either only CWI [12, 13] or IWI [14-16], which precluded the detection of different pathogeneses for different types and subtypes of WSI. Moreover, chronic cerebral infarction was not ruled out in many previous studies due to the absence of DWI sequence. Furthermore, the concurrence of both CWI and IWI, which is defined as mixed-type in our study, has not been included in any previous studies to our knowledge.

So far, only five studies [3, 5, 17-19] have explicitly examined the pathophysiological differences of CWI and IWI, but three of them did not use DWI sequence and two of them had selection bias for enrolling patients with cerebrovascular stenosis or occlusion.

The results of our study demonstrated an association between IWI and critical stenosis of ICA, whereas the association is weakest in CWI patients. Consistent with previous research [14], these findings supported the theory that HDI may be the main cause of IWI. The relationship between CWI and HDI appears more complicated with previous research stating that [18] artery-to-artery embolism might play an important role in isolated CWI.

Table 2 Clinical course and prognosis at 90th day of patients with different types of WSI

\begin{tabular}{lllll}
\hline Variable & $\begin{array}{l}\text { CWI } \\
(n=92)\end{array}$ & $\begin{array}{l}\text { IWI } \\
(n=112)\end{array}$ & $\begin{array}{l}\text { Mixed type } \\
(n=136)\end{array}$ & $P$ \\
\hline $\begin{array}{l}\text { Initial NIHSS Score } \\
\text { Clinical course, } n(\%)\end{array}$ & $3(1,5)$ & $3(1,6)$ & $3(1,5)$ & 0.423 \\
$\quad$ Improving & $33(35.9)$ & $18(16.1)$ & $26(19.1)$ & 0.002 \\
$\quad$ Stable & $50(54.3)$ & $56(50.0)$ & $76(55.9)$ & 0.641 \\
$\quad \begin{array}{l}\text { Deteriorated } \\
\text { Prognosis at 90th day }\end{array}$ & $12(13.0)^{\text {a }}$ & $38(33.9)^{\text {a }}$ & $35(25.7)$ & 0.003 \\
$\quad$ & & & \\
Poor outcome, $n(\%)$ & $21(22.8)^{\text {a }}$ & $47(42.0)^{\text {a }}$ & $50(36.8)$ & 0.014 \\
\hline
\end{tabular}

Data are presented as counts (\%)

WSI Watershed infarction, CWI Cortical watershed infarction, IWI Internal watershed infarction, NIHSS The National Institutes of Health Stroke Scale, $N$ number of persons

a Significant difference between CWI and IWI group
The susceptibility of internal border-zone area to HDI is probably due to low perfusion pressure in perforating medullary arteries, the most distal branches of the ICA with insufficient collateral supply of the deep perforating lenticulostriate arties.

To the best of our knowledge, no existing research has directly compared the hemodynamics of CWI subtypes. The available data [20] suggest that HDI is more frequently documented in the anterior border-zone area. Furthermore, for CWI concurrent with IWI, the AWI subtype had a higher incidence than the PWI subtype [21]. We found that AWI was prone to association with the critical stenosis of ICA. The vulnerability of the anterior border-zone area may be attributed to the fact that MCA and ACA are supplied only by ICA; thus stenosis or occlusion of ICA will cause AWI, and inefficient collateralization will further worsen the perfusion. Conversely, severe stenosis or occlusion of the vertebrobasilar system, or a fetal-type PCA may be responsible for PWI, which is probably rarer than the former.

A previous study [18] revealed that the rosary-like infarction in the centrum semiovale, which was identified as P-IWI in our study, appeared to be associated with HDI. But Moustafa RR et al. [22] found that in addition to HDI, microemboli might also play a role in the pathogenesis of rosary-like infarction. But few studies have compared the difference between C-IWI and P-IWI precisely. We found that critical ICA stenosis was more common in P-IWI patients than in C-IWI patients. This was contrary to a previous study [16] which identified 14 C-IWI and 13 P-IWI and found that occlusive diseases of ICA were more prevalent in C-IWI, but it was highly likely to be limited by the small sample size. Internal border-zone area is located in the junction of the ACA and MCA superficial perforators, a special anatomical location that shapes its sensitivity to hypoperfusion. Thus, the centrum semiovale, which represents the most distal region perfused by the ICA, is most vulnerable to HDI. 
We demonstrated that patients with IWI had worse hospital courses and poor prognosis 3 months post stroke, and that IWI was an independent risk factor for poor outcome. A previous study [15] also identified IWI as a predictor of early neurological deterioration in minor stroke patients. These results suggest that more intensive care is necessary for patients with IWI, especially P-IWI, so as to improve perfusion.

Our study is also limited for the following reasons. Firstly, the number of patients in each subtype is relatively small although our study is already the largest in scale to date. Secondly, the location of border-zone is sometimes hard to define because vascular territories may vary greatly among individuals. Thirdly, our study was undertaken in the Chinese population in whom MCA stenosis is more prevalent, but there was no significant difference of the incidence of significant MCA stenosis or occlusion among 3 types of WSI, which need to be further studied.

\section{Conclusion}

IWI, the P-IWI subtype in particular, seems to be related to HDI and poor prognosis, whereas CWI shows a weaker correlation with ICA steno-occlusion. Better understanding of the different pathogenesis of WSI would help identify patients at risk and prevent clinical deterioration.

\section{Additional file}

Additional file 1: Table S1. Incidence of critical cerebrovascular stenosis in different WSI types. (DOCX $17 \mathrm{~kb}$ )

\begin{abstract}
Abbreviations
ACA: Anterior cerebral artery; ANOVA: One-way analysis of variance; AWl: Anterior watershed infarction; BP: Blood pressure; CAD: Coronary artery disease; C-IWI: Confluent IWl; Cr: Creatinine; CTA: Computed tomographic angiogram; CWI: Cortical watershed infarction; DBP: Diastolic blood pressure; DWl: Diffusion-weighted imaging; Fib: Fibrinogen; HbA1c: Glycosylated hemoglobin; HCY: Homocysteine; HDl: Hemodynamic impairment; HDL: High density lipoprotein; hs-CRP: High-sensitivity C-reactive protein; ICA: Internal carotid artery; IWI: Internal watershed infarction; LDL: Low density lipoprotein; MCA: Middle cerebral artery; MRI: Magnetic resonance imaging; mRS: Modified Rankin Scale; NIHSS: The National Institutes of Health Stroke Scale; OR: odds ratio; PCA: Posterior cerebral artery; P-IWI: Partial IWI; PWI: Posterior watershed infarction; SBP: Systolic blood pressure; SD: Standard deviation; TC: total cholesterol; TG: Triglyceride; TIA: Transient ischemic attack; UA: Uric acid; WSI: Watershed infarction
\end{abstract}

\section{Acknowledgements}

Not applicable.

\section{Funding}

This study was supported by National Natural Science Foundation of China (Grant No 81271309).

\section{Availability of data and materials}

The datasets used in the current study are available from the corresponding author upon reasonable request.

\section{Authors' contributions}

YL planned the study, collected data, and wrote the manuscript. XYZ and WQ collected the data and revised the manuscript. ML and LY analyzed the data and revised the manuscript. SY, HF and JY interpreted the data and revised the manuscript. WLH designed the study and revised the manuscript. All authors read and approved the final manuscript.

\section{Ethics approval and consent to participate}

This study was approved by the Ethics Committee of Beijing Chao-Yang Hospital, Capital Medical University and all participants gave written informed consent for participation and to publication.

\section{Consent for publication}

All patients agreed with the publication.

\section{Competing interests}

On behalf of all authors, the corresponding author states that there is no competing interest.

\section{Publisher's Note}

Springer Nature remains neutral with regard to jurisdictional claims in published maps and institutional affiliations.

\section{Author details}

${ }^{1}$ Department of Neurology, Beijing Chao-Yang Hospital, Capital Medical University, No. 8 South Gongti Road, Chaoyang district, Beijing 100020, China. ${ }^{2}$ Department of Radiology, Beijing Chao-Yang Hospital, Capital Medical University, Beijing, China. ${ }^{3}$ Department of Neurology, Qianfoshan Hospital, Shandong University, Jinan, China.

Received: 11 April 2017 Accepted: 21 August 2017

Published online: 29 August 2017

References

1. Hoffman SJ, Yee AH, Slusser JP, Rihal CS, Holmes DR Jr, Rabinstein AA, Gulati R. Neuroimaging patterns of ischemic stroke after percutaneous coronary intervention. Catheter Cardiovasc Interv. 2015;85(6):1033-40.

2. Wong KS, Gao S, Chan YL, Hansberg T, Lam WW, Droste DW, Kay R, Ringelstein EB. Mechanisms of acute cerebral infarctions in patients with middle cerebral artery stenosis: a diffusion-weighted imaging and microemboli monitoring study. Ann Neurol. 2002;52(1):74-81.

3. Bisschops RH, Klijn CJ, Kappelle LJ, van Huffelen AC, van der Grond J. Association between impaired carbon dioxide reactivity and ischemic lesions in arterial border zone territories in patients with unilateral internal carotid artery occlusion. Arch Neurol. 2003;60(2):229-33.

4. Arakawa S, Minematsu K, Hirano T, Tanaka Y, Hasegawa Y, Hayashida K, Yamaguchi T. Topographic distribution of misery perfusion in relation to internal and superficial borderzones. AJNR Am J Neuroradiol. 2003; 24(3):427-35

5. Yong SW, Bang OY, Lee PH, Li WY. Internal and cortical border-zone infarction: clinical and diffusion-weighted imaging features. Stroke. 2006; 37(3):841-6.

6. Kim DE, Lee KB, Roh H, Ahn MY, Roh JK. Association of internal border zone infarction with middle cerebral artery steno-occlusion. Eur Neurol. 2010; 64(3):178-85.

7. Mangla R, Kolar B, Almast J, Ekholm SE. Border zone infarcts: pathophysiologic and imaging characteristics. Radiographics. 2011;31(5):1201-14.

8. Samuels OB, Joseph GJ, Lynn MJ, Smith HA, Chimowitz MI. A standardized method for measuring intracranial arterial stenosis. AJNR Am J Neuroradiol. 2000;21(4):643-6.

9. Ogata J, Yutani C, Otsubo R, Yamanishi H, Naritomi H, Yamaguchi T, Minematsu K. Heart and vessel pathology underlying brain infarction in 142 stroke patients. Ann Neurol. 2008;63(6):770-81.

10. Caplan LR, Hennerici M. Impaired clearance of emboli (washout) is an important link between hypoperfusion, embolism, and ischemic stroke. Arch Neurol. 1998;55(11):1475-82.

11. Moustafa RR, Izquierdo-Garcia D, Jones PS, Graves MJ, Fryer TD, Gillard JH, Warburton EA, Baron JC. Watershed infarcts in transient ischemic attack/ minor stroke with $>$ or $=50 \%$ carotid stenosis: hemodynamic or embolic? Stroke. 2010;41(7):1410-6. 
12. Nasel C, Azizi A, Wilfort A, Mallek R, Schindler E. Measurement of time-topeak parameter by use of a new standardization method in patients with stenotic or occlusive disease of the carotid artery. AJNR Am J Neuroradiol. 2001;22(6):1056-61.

13. Joinlambert C, Saliou G, Flamand-Roze C, Masnou P, Sarov M, Souillard R, Saliou-Theaudin M, Guedj T, Assayag P, Ducreux D, et al. Cortical borderzone infarcts: clinical features, causes and outcome. J Neurol Neurosurg Psychiatry. 2012;83(8):771-5.

14. Del Sette M, Eliasziw M, Streifler JY, Hachinski VC, Fox AJ, Barnett HJM. Internal Borderzone Infarction: A Marker for Severe Stenosis in Patients With Symptomatic Internal Carotid Artery Disease. Stroke. 2000;31(3):631-6.

15. Kim JT, Yoon GJ, Nam TS, Choi SM, Lee SH, Park MS, Kim BC, Kim MK, Cho $\mathrm{KH}$. Internal border zone lesions as a predictor of early neurological deterioration in minor stroke patients with severe arterial steno-occlusion. J Neuroimaging. 2011;21(2):173-6.

16. Li HF, Zhang X, Zhang Y, Pan XD, Zhao HQ, Li H. Clinical and neuroradiological features of internal watershed infarction and the occlusive diseases of carotid artery system. Neurol Res. 2010;32(10):1090-6.

17. Moriwaki H, Matsumoto M, Hashikawa K, Oku N, Ishida M, Seike Y, Watanabe $Y$, Hougaku $H$, Handa N, Nishimura T. Hemodynamic aspect of cerebral watershed infarction: assessment of perfusion reserve using iodine123-iodoamphetamine SPECT. J Nucl Med. 1997;38(10):1556-62.

18. Derdeyn CP, Khosla A, Videen TO, Fritsch SM, Carpenter DL, Grubb RL Jr, Powers WJ. Severe hemodynamic impairment and border zone-region infarction. Radiology. 2001;220(1):195-201.

19. Sorgun MH, Rzayev S, Yilmaz V, Isikay CT. Etiologic Subtypes of Watershed Infarcts. J Stroke Cerebrovasc Dis. 2015;24(11):2478-83.

20. Wiart M, Berthezene Y, Adeleine P, Feugier P, Trouillas P, Froment JC, Nighoghossian $\mathrm{N}$. Vasodilatory response of border zones to acetazolamide before and after endarterectomy : an echo planar imaging-dynamic susceptibility contrast-enhanced MRI study in patients with high-grade unilateral internal carotid artery stenosis. Stroke. 2000;31(7):1561-5.

21. Krapf H, Widder B, Skalej M. Small rosarylike infarctions in the centrum ovale suggest hemodynamic failure. AJNR Am J Neuroradiol. 1998;19(8):1479-84.

22. Moustafa RR, Momjian-Mayor I, Jones PS, Morbelli S, Day DJ, Aigbirhio Fl, Fryer TD, Warburton EA, Baron JC. Microembolism versus hemodynamic impairment in rosary-like deep watershed infarcts: a combined positron emission tomography and transcranial Doppler study. Stroke. 2011;42(11):3138-43.

\section{Submit your next manuscript to BioMed Central and we will help you at every step:}

- We accept pre-submission inquiries

- Our selector tool helps you to find the most relevant journal

- We provide round the clock customer support

- Convenient online submission

- Thorough peer review

- Inclusion in PubMed and all major indexing services

- Maximum visibility for your research

Submit your manuscript at wuw biomedcentral.com/submit

) Biomed Central 